\title{
Targeted next-generation sequencing in the detection of mismatch repair deficiency in endometrial cancers
}

\author{
Fei Dong ${ }^{1} \cdot$ Danielle C. Costigan ${ }^{1} \cdot$ Brooke E. Howitt $^{2}$
}

Received: 6 June 2018 / Revised: 27 July 2018 / Accepted: 3 August 2018 / Published online: 11 September 2018

(c) United States \& Canadian Academy of Pathology 2018

\begin{abstract}
Mismatch repair deficiency represents a biomarker of immunotherapy response and a phenotypic feature of Lynch syndrome-associated endometrial cancers. Using a targeted next-generation sequencing assay, we identified molecular features of mismatch repair deficiency, specifically insertion and deletion mutations in mononucleotide repeats, and established thresholds for the number of such mutations to classify endometrial cancers as mismatch repair deficient, proficient, or indeterminate. Sequencing classification was compared to the loss of MLH1, MSH2, MSH6, or PMS2 expression by immunohistochemistry. A total of 259 endometrial cancers were classified by sequencing as mismatch repair deficient $(n=48,19 \%)$, proficient $(n=199,77 \%)$, or indeterminate $(n=12,5 \%)$. Sequencing findings were concordant with loss of expression of at least one mismatch repair protein in 47 of $48(98 \%)$ cases classified as deficient and retained expression of all four proteins in 190 of $199(95 \%)$ cases classified as proficient. Of the 12 cases classified as indeterminate, $7(58 \%)$ demonstrated mismatch repair protein loss. Overall, targeted next-generation sequencing exhibited a high rate of concordance with immunohistochemistry for mismatch repair deficiency; however, sequencing was indeterminate in a few cases and demonstrated a false negative rate of 5\%. Although we recommend implementation of a mismatch repair deficiency algorithm for laboratories performing next-generation sequencing cancer panels, immunohistochemistry remains a cost-effective screening method for mismatch repair deficiency in endometrial cancer.
\end{abstract}

\section{Introduction}

Mismatch repair deficiency is characterized by loss of expression of one or more mismatch repair proteins MLH1, MSH2, MSH6, and PMS2 and is a feature of approximately $20 \%$ of endometrial cancers [1-8]. The consequences of defective mismatch repair machinery include hypermutation and insertion and deletion errors in genomic DNA repeats, leading to microsatellite instability. Mismatch repair deficiency is a defining feature of the microsatellite instability molecular subclass of endometrial cancers as classified by the Cancer Genome Atlas project [9].

Brooke E. Howitt

bhowitt@stanford.edu

1 Department of Pathology, Brigham and Women's Hospital, Boston, MA, USA

2 Department of Pathology, Stanford University Medical Center, Stanford, CA, USA
The most common mechanism of mismatch repair deficiency in sporadic endometrial cancer is via epigenetic silencing of $M L H 1$ by promoter hypermethylation [10,11]. Mismatch repair deficiency may also result from germline or somatic mutation of the mismatch repair genes, and pathogenic germline variants establish a diagnosis of Lynch syndrome, which predisposes patients to increased risk of cancers of colorectal, endometrial, and other sites [12].

Recently, mismatch repair deficiency has been demonstrated to be an important biomarker for therapy selection with immune checkpoint inhibitors. Endometrial cancers with microsatellite instability have been associated with elevated mutational burden, increased tumor-infiltrating lymphocytes, and elevated programmed cell death protein1 (PD-1) and programmed death-ligand 1 (PD-L1) expression in tumor associated lymphocytes [13-15]. Mismatch repair deficiency has been associated with clinical response to immunotherapy in multiple tumor types [16], and in May 2017, the Food and Drug Administration granted accelerated approval of pembrolizumab for advanced solid tumors with mismatch repair deficiency or microsatellite instability, regardless of pathological diagnosis [17]. 
In addition to identifying driver mutations, broad cancer sequencing panels can use sequencing data to identify patterns within incidentally sequenced passenger mutations. Our laboratory and others have developed next-generation sequencing algorithms to detect phenotypic features of mismatch repair deficiency and microsatellite instability from cancer panel sequencing data [18-21]. These metrics have demonstrated high concordance with mismatch repair protein immunohistochemistry and microsatellite analysis by polymerase chain reaction in colorectal cancer.

Since colorectal cancer is the most common cancer type with mismatch repair deficiency, most sequencing algorithms are validated predominantly on this cancer type, and the efficacy of sequencing-based detection of mismatch repair deficiency in endometrial cancers is not as well established. Endometrial cancers are biologically different than colorectal cancers, and endometrial cancers with mismatch repair deficiency acquire mutations in different microsatellite regions compared to colorectal cancers [22]. Therefore, we hypothesize that any mismatch repair deficiency detection algorithm trained on colorectal cancers needs to be adjusted to improve accuracy in endometrial cancers.

Here, we test our algorithm for mismatch repair deficiency detection in a cohort of endometrial cancers. Through this study, we hope to better understand the molecular phenotype of endometrial cancers with mismatch repair deficiency and the utility of next-generation sequencing to inform clinical decision making.

\section{Materials and methods}

\section{Patient selection and enrollment}

Study volunteers were prospectively enrolled via Profile, an institutional study for cancer genotyping, and provided written consent for cancer sequencing [23]. Subjects provided written consent at the Dana Farber Cancer Institute. Sequencing and immunohistochemistry were performed at Brigham and Women's Hospital. Universal screening for MLH1, MSH2, MSH6, and PMS2 protein expression was clinically performed for endometrial cancers at our institution [1]. Endometrial cancers that had undergone targeted next-generation sequencing were identified and matched to corresponding surgical pathology reports. A total of 259 patients who had both sequencing and screening by immunohistochemistry were included in the study. Pathological diagnoses included endometrioid (184), serous (39), clear cell (5), undifferentiated (7), mixed (7), and unclassified high-grade Mullerian carcinoma (1) and carcinosarcoma (16). This project was approved by the Dana
Farber Cancer Institute Institutional Review Board and the Partners Human Research Committee.

\section{Mismatch repair immunohistochemistry}

Immunohistochemistry was conducted on 4- $\mu$ m-thick formalin-fixed, paraffin-embedded tissue sections using mouse anti-MLH1 monoclonal antibody, mouse anti-MSH2 monoclonal antibody, mouse anti-PMS2 monoclonal antibody, and mouse anti-MSH6 monoclonal antibody using the Envision Plus Detection System, as previously described [20]. Briefly, the following antibodies and dilutions were applied: (1) MSH2 monoclonal antibody (Cal Biochem- EMD Millipore, clone NA27) 1:200; (2) MSH6 monoclonal antibody (BD Bioscience, clone PU29) 1:50; (3) MLH1 monoclonal antibody (Novocastra, clone NCLL-MLH1) 1:75; and (4) PMS2 monoclonal antibody (Cell Marque, clone MRQ-28) 1:100. The antigen retrieval method for all stains included heat-induced epitope retrieval in either $\mathrm{pH} 6.110 \mathrm{mM}$ citrate buffer (MSH6, MLH1, PMS2) or pH 8.0 EDTA (MSH2), with a pressure cooker. Internal positive controls (stroma, vasculature) and appropriate negative controls were used.

\section{Targeted next-generation sequencing}

Targeted next-generation sequencing was performed using OncoPanel, which has been previously described [24]. In brief, DNA was isolated from formalin-fixed, paraffinembedded or frozen, optimal cutting temperature-embedded tissue in regions with at least $20 \%$ cancer cell nuclei. At least $50 \mathrm{ng}$ DNA was used for library preparation, and hybrid capture with a custom RNA bait set by Agilent SureSelect (Agilent Technologies, Santa Clara, CA) was performed to enrich for exons of cancer-associated genes. Three versions of the assay were used, depending on the time of enrollment, including coding regions of 275, 298, or 447 genes encompassing 757,787, 831,033, or 1,315,708 bp of the genome, respectively. Thus, 39 specimens were tested with version 1,170 were tested with version 2 , and 50 were tested with version 3. Sequencing was performed on the HiSeq 2500 System (Illumina, San Diego, CA). Informatics was performed using a custom pipeline, and insertion and deletion mutations were called using Indelocator (Broad Institute, Cambridge, MA).

\section{Mismatch repair deficiency detection algorithm}

The mismatch repair algorithm identified single-nucleotide insertion and deletion events in DNA mononucleotide repeat regions of at least four consecutive nucleotides and were designated mismatch repair deficiency-associated 


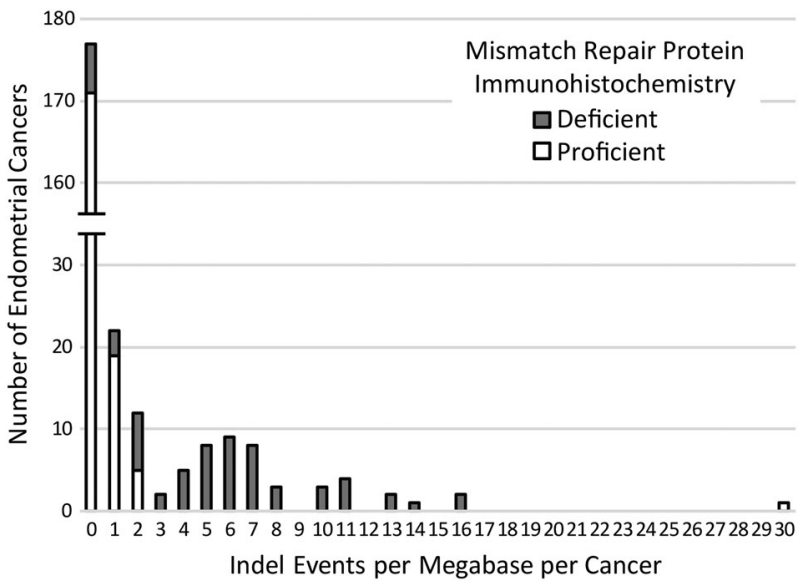

Fig. 1 Distribution of mismatch repair-deficient and proficient endometrial cancers with respect to sequencing findings. Mismatch repair deficiency is assessed by immunohistochemical expression of MLH1, MSH2, MSH6, and PMS2. The $x$-axis shows the number of insertion and deletion events in mononucleotide repeats per megabase per case by sequencing, rounded to the nearest integer. Each bar represents the number of mismatch repair-deficient or -proficient endometrial cancers with that number of events

events. Such events were previously demonstrated to be enriched in colorectal cancers with mismatch repair deficiency [21]. The total number of mismatch repair deficiency-associated events were normalized to the size of the panel to establish the number of events per megabase (indel/Mb) for each case. Indel/Mb values were compared to immunohistochemistry results to establish thresholds for mismatch-deficient, -proficient, and -indeterminate cancers by sequencing.

The accuracy of sequencing for the detection of mismatch repair deficiency was calculated as follows: sensitivity, the percent of all cases with loss of expression of at least one mismatch repair protein by immunohistochemistry that is classified as mismatch repair deficient by sequencing; specificity, the percent of all cases with retained expression of all mismatch repair proteins by immunohistochemistry that is classified as mismatch repair proficient by sequencing; positive predictive value, the percent of all cases classified as mismatch repair deficient by sequencing that has loss of at least one mismatch repair protein by immunohistochemistry; and negative predictive value, the percent of all cases classified as mismatch repair proficient that has retained expression of all mismatch repair proteins by immunohistochemistry.

\section{Statistical analysis}

Two-sided Mann-Whitney $U$ test was used to compare distributions of continuous variables with significance set at $P<0.05$.

\section{Results}

\section{Insertion and deletion events in mismatch repair- deficient endometrial cancers}

The number of mismatch repair deficiency-associated events per megabase for each endometrial cancer was calculated. The distribution of the number of events in mismatch repair-deficient and mismatch repair-proficient endometrial cancers, as determined by immunohistochemistry, is shown in Fig. 1.

Of the 259 endometrial cancers in the study, 63 (24\%) had loss of immunohistochemical expression of at least one mismatch repair protein. The median number of mismatch repair deficiency-associated events was 6.0 indel/ $\mathrm{Mb}$ (mean 5.8, standard deviation 4.0, range 0.0-16.0) for mismatch repair-deficient cancers compared to 0.0 indel $/ \mathrm{Mb}$ (mean 0.3 , standard deviation 2.2, range 0.0-29.6) for mismatch repair-proficient cancers $(P<0.0001)$.

Of the 63 endometrial cancers with mismatch repair deficiency, 47 (74.6\%) had $>2.5$ indel/Mb by sequencing, and 54 of $63(85.7 \%)$ endometrial cancers with mismatch repair deficiency had $>1.5$ indel/Mb by sequencing. In contrast, 171 of 196 (87.2\%) endometrial cancers with intact immunohistochemical staining had no mismatch repair deficiency-associated events detected, and 190 of 196 (96.9\%) had $<1.5$ indel/Mb by sequencing.

\section{Concordance of sequencing interpretation with immunohistochemistry}

Using the distribution described in Fig. 1, a single threshold of 1.5 indel/Mb achieved the highest degree of concordance of $94 \%$ between sequencing and immunohistochemistry, with agreement in 244 of 259 cases. Of the 63 cancers with loss of expression of at least one mismatch repair protein by immunohistochemistry, 54 had greater than 1.5 indel/Mb by sequencing (sensitivity 54/63, 86\%). Of the 196 cancers with intact expression of all mismatch repair proteins by immunohistochemistry, 190 had less than 1.5 indel/Mb mismatch repair proficient by sequencing (specificity 190/ 196, 97\%).

However, we recognized that cancers with 1.5 to 2.5 indel/Mb were difficult to classify, with 7 of 12 cases in this group exhibiting mismatch repair protein loss by immunohistochemistry. Therefore, we further divided our cohort into three categories based on sequencing interpretation: 'deficient' ( $>2.5$ indel/Mb), 'proficient' $(<1.5$ indel $/ \mathrm{Mb})$, and 'indeterminate' $(1.5-2.5$ indel/Mb). Of the 48 cancers predicted to be deficient by sequencing, 47 had loss of expression of a mismatch repair protein by immunohistochemistry (positive predictive value $47 / 48,98 \%$ ). Of the 199 cancers predicted to be proficient by sequencing, 190 
Table 1 Next-generation sequencing determination of mismatch repair deficiency, defined by number of indel events in mononucleotide repeats, compared to immunohistochemistry, defined by loss of nuclear expression of MLH1, MSH2, MSH6, or PMS2

\begin{tabular}{llll}
\hline & & $\begin{array}{l}\text { Immunohistochemistry } \\
\text { Deficient (protein loss) }\end{array}$ & Proficient (proteins intact) \\
\hline \multirow{2}{*}{ Sequencing } & Deficient $(>2.5$ indel/Mb) & 47 & 1 \\
& Proficient $(<1.5$ indel $/ \mathrm{Mb})$ & 9 & 190 \\
& Indeterminate $(1.5-2.5$ indel/Mb) & 7 & 5 \\
\hline
\end{tabular}

had retained expression of mismatch repair proteins by immunohistochemistry (negative predictive value 190/199, $95 \%$, Table 1).

\section{Review of discordant cases}

The only cancer predicted to be deficient by sequencing with intact mismatch repair protein expression was an undifferentiated carcinoma with the highest number of mismatch repair deficiency-associated events (29.6 indel/ $\mathrm{Mb}$ ) in the cohort. Sequencing identified a POLE p.V411L mutation and an elevated number of single-nucleotide variants, and the phenotype is consistent with a POLE-associated ultramutated endometrial cancer.

Of the nine cases predicted to be proficient by sequencing with loss of at least one mismatch repair protein expression, the discordance may be due to several factors. Two of the nine cancers exhibited isolated loss of MSH6 expression. Three of the nine cancers with mismatch repair deficiency missed by sequencing showed low variant allele fractions of less than $10 \%$ for pathogenic somatic mutations, consistent with specimens with low tumor purity. No definitive explanation for discordance between sequencing and immunohistochemistry could be identified for the remaining four specimens.

\section{Discussion}

Phenotypic mismatch repair deficiency can be assessed in the pathology laboratory through immunohistochemical evaluation for nuclear protein expression in tumor cells. Although phenotypic screening for Lynch syndrome by immunohistochemistry is universally recommended for colorectal cancer in consensus guidelines [25], such guidelines do not exist for endometrial cancer [26]. However, studies have suggested the added value of universal Lynch syndrome screening in patients with endometrial cancer [1, 3, 5, 27].

As sequencing costs have declined, multiple cancer panel next-generation sequencing assays have been developed to profile genomic alterations for clinical use [24, 28-30]. These assays aim to identify driver events in cancer development to help guide therapy selection and are major decision points in multi-institutional basket trials for targeted cancer therapy [31, 32]. As next-generation sequencing technology is rapidly adopted into clinical practice in molecular laboratories [33], it is rational to use a single set of sequencing data for multiple clinical indications, including screening for mismatch repair deficiency.

We have previously described an algorithm counting mismatch repair deficiency-associated events and ultimately demonstrated that this simple metric achieved 96\% sensitivity and $99 \%$ specificity compared to mismatch repair immunohistochemistry in colorectal cancer [21]. The application of a similar metric in endometrial cancer demonstrates a poorer level of performance in this study, achieving at best $86 \%$ sensitivity and $97 \%$ specificity with a false negative rate of $5 \%$ in our cohort.

Potential explanations for false negative results include a higher frequency of endometrial cancers with isolated MSH6 loss. Germline MSH6 mutations are known to confer a higher risk for endometrial cancers compared to colorectal cancers [34], and affected cancers frequently exhibit microsatellite instability-low status [35]. Another potential limitation is low tumor purity in endometrial cancer specimens, which are susceptible to contamination with nonneoplastic cells from endometrial stroma and myometrium.

Mismatch repair deficiency is known to confer different molecular phenotypes in endometrial cancers compared to colorectal cancers. Before the invention of next-generation sequencing technology, investigators recognized that some recurrent microsatellite alterations in colorectal cancers were not seen in endometrial cancers with mismatch repair deficiency [36]. Analysis of targeted exonic microsatellites demonstrated fewer mutations in endometrial cancers compared to colorectal cancers with mismatch repair deficiency [37].

Similar findings have since been described in wholeexome sequencing data. The only paper to perform extensive direct comparisons between mismatch repair-deficient colorectal and endometrial cancers used the Cancer Genome Atlas data to demonstrate that colorectal cancers had more microsatellite instability events compared to endometrial cancer [22]. Using a cutoff of greater than 50 events, the authors' methodology achieved $100 \%$ accuracy in distinguishing microsatellite instability-high versus microsatellite instability-low or microsatellite stable colorectal cancer but 
only $83 \%$ sensitivity ( 25 of 30 ) and $97 \%$ specificity (97 of $100)$ in endometrial cancer. These figures are comparable to our current classification using a much more limited targeted panel. Another article evaluating the correlation between whole-exome sequencing data and microsatellite instability across many cancer types demonstrated discordance in 11 of 171 microsatellite instability-high cancers, of which 10 of 11 discordant cases were endometrial cancers [38].

Our current findings and the literature highlight phenotypic differences in genomic alterations across tumor types as a result of mismatch repair deficiency. The unique biology and molecular phenotype of endometrial cancers may limit accurate detection of mismatch repair deficiency in this disease compared to colorectal cancer, and algorithms using next-generation sequencing data to broadly screen for mismatch repair deficiency may need to be modified for endometrial cancer. In the setting of routine clinical screening, immunohistochemistry remains an accurate and cost-effective method for mismatch repair deficiency screening in endometrial cancer.

Despite these limitations, our algorithm applied to a limited targeted cancer gene panel still performs well with 94.2\% concordance compared to immunohistochemistry. As targeted next-generation sequencing becomes available for clinical cancer care, the ability to obtain more actionable information, including determination of mismatch repair deficiency, from sequencing data will help increase clinical benefit relative to sequencing cost. Based on these results and prior work [20, 21], we have since adopted this methodology to screen for mismatch repair deficiency in all cancers undergoing next-generation sequencing in our laboratory, and we encourage other laboratories to explore similar validations in endometrial cancers and other cancer types.

\section{Compliance with ethical standards}

Conflict of interest The authors declare that they have no conflict of interest.

\section{References}

1. Watkins JC, Yang EJ, Muto MG, Feltmate CM, Berkowitz RS, Horowitz NS, et al. Universal screening for mismatch-repair deficiency in endometrial cancers to identify patients with Lynch syndrome and Lynch-like syndrome. Int $\mathrm{J}$ Gynecol Pathol. 2017;36:115-27.

2. Stelloo E, Jansen AML, Osse EM, Nout RA, Creutzberg CL, Ruano D, et al. Practical guidance for mismatch repair-deficiency testing in endometrial cancer. Ann Oncol. 2017;28:96-102.

3. Mills AM, Liou S, Ford JM, Berek JS, Pai RK, Longacre TA. Lynch syndrome screening should be considered for all patients with newly diagnosed endometrial cancer. Am J Surg Pathol. 2014;38:1501-9.

4. Moline J, Mahdi H, Yang B, Biscotti C, Roma AA, Heald B, et al. Implementation of tumor testing for Lynch syndrome in endometrial cancers at a large academic medical center. Gynecol Oncol. 2013;130:121-6.

5. Ferguson SE, Aronson M, Pollett A, Eiriksson LR, Oza AM, Gallinger $\mathrm{S}$, et al. Performance characteristics of screening strategies for Lynch syndrome in unselected women with newly diagnosed endometrial cancer who have undergone universal germline mutation testing. Cancer. 2014;120:3932-9.

6. Hampel H, Frankel W, Panescu J, Lockman J, Sotamaa K, Fix D, et al. Screening for Lynch syndrome (hereditary nonpolyposis colorectal cancer) among endometrial cancer patients. Cancer Res. 2006;66:7810-7.

7. Leenen CHM, van Lier MGF, van Doorn HC, van Leerdam ME, Kooi SG, de Waard J, et al. Prospective evaluation of molecular screening for Lynch syndrome in patients with endometrial cancer $\leq 70$ years. Gynecol Oncol. 2012;125:414-20.

8. Dillon JL, Gonzalez JL, DeMars L, Bloch KJ, Tafe LJ. Universal screening for Lynch syndrome in endometrial cancers: frequency of germline mutations and identification of patients with Lynchlike syndrome. Hum Pathol. 2017;70:121-8.

9. Cancer Genome Atlas Research Network, Kandoth C, Schultz N, Cherniack AD, Akbani R, Liu Y, et al. Integrated genomic characterization of endometrial carcinoma. Nature. 2013;497:67-73.

10. Esteller M, Levine R, Baylin SB, Ellenson LH, Herman JG. MLH1 promoter hypermethylation is associated with the microsatellite instability phenotype in sporadic endometrial carcinomas. Oncogene. 1998;17:2413-7.

11. Simpkins SB, Bocker T, Swisher EM, Mutch DG, Gersell DJ, Kovatich AJ, et al. MLH1 promoter methylation and gene silencing is the primary cause of microsatellite instability in sporadic endometrial cancers. Hum Mol Genet. 1999;8:661-6.

12. Lynch HT, Snyder CL, Shaw TG, Heinen CD, Hitchins MP. Milestones of Lynch syndrome: 1895-2015. Nat Rev Cancer. 2015;15:181-94.

13. Howitt BE, Shukla SA, Sholl LM, Ritterhouse LL, Watkins JC, Rodig $\mathrm{S}$, et al. Association of polymerase e-mutated and microsatellite-instable endometrial cancers with neoantigen load, number of tumor-infiltrating lymphocytes, and expression of PD-1 and PD-L1. JAMA Oncol. 2015;1:1319-23.

14. Sloan EA, Ring KL, Willis BC, Modesitt SC, Mills AM. PD-L1 expression in mismatch repair-deficient endometrial carcinomas, including Lynch syndrome-associated and MLH1 promoter hypermethylated tumors. Am J Surg Pathol. 2017;41:326-33.

15. Eggink FA, Van Gool IC, Leary A, Pollock PM, Crosbie EJ, Mileshkin L, et al. Immunological profiling of molecularly classified high-risk endometrial cancers identifies POLE-mutant and microsatellite unstable carcinomas as candidates for checkpoint inhibition. Oncoimmunology. 2017;6:e1264565.

16. Le DT, Durham JN, Smith KN, Wang H, Bartlett BR, Aulakh LK, et al. Mismatch repair deficiency predicts response of solid tumors to PD-1 blockade. Science. 2017;357:409-13.

17. Lemery S, Keegan P, Pazdur R, First FDA Approval agnostic of cancer site - when a biomarker defines the indication. N Engl J Med. 2017;377:1409-12.

18. Salipante SJ, Scroggins SM, Hampel HL, Turner EH, Pritchard CC. Microsatellite instability detection by next generation sequencing. Clin Chem. 2014;60:1192-9.

19. Stadler ZK, Battaglin F, Middha S, Hechtman JF, Tran C, Cercek A, et al. Reliable detection of mismatch repair deficiency in colorectal cancers using mutational load in next-generation sequencing panels. J Clin Oncol. 2016;34:2141-7. 
20. Nowak JA, Yurgelun MB, Bruce JL, Rojas-Rudilla V, Hall DL, Shivdasani $\mathrm{P}$, et al. Detection of mismatch repair deficiency and microsatellite instability in colorectal adenocarcinoma by targeted next-generation sequencing. J Mol Diagn. 2017;19:84-91.

21. Papke DJ, Nowak JA, Yurgelun MB, Frieden A, Srivastava A, Lindeman NI, et al. Validation of a targeted next generation sequencing approach to detect mismatch repair deficiency in colorectal adenocarcinoma [published online 28 June 2018]. Mod Pathol. https://doi.org/10.1038/s41379-018-0091-x

22. Kim T-M, Laird PW, Park PJ. The landscape of microsatellite instability in colorectal and endometrial cancer genomes. Cell. 2013;155:858-68.

23. MacConaill LE, Garcia E, Shivdasani P, Ducar M, Adusumilli R, Breneiser $\mathrm{M}$, et al. Prospective enterprise-level molecular genotyping of a cohort of cancer patients. $\mathrm{J}$ Mol Diagn. 2014;16:660-72.

24. Garcia EP, Minkovsky A, Jia Y, Ducar MD, Shivdasani P, Gong $\mathrm{X}$, et al. Validation of OncoPanel: a targeted next-generation sequencing assay for the detection of somatic variants in cancer. Arch Pathol Lab Med. 2017;141:751-8.

25. Giardiello FM, Allen JI, Axilbund JE, Boland CR, Burke CA, Burt RW, et al. Guidelines on genetic evaluation and management of Lynch syndrome: a consensus statement by the US Multisociety Task Force on colorectal cancer. Am J Gastroenterol. 2014;109:1159-79.

26. Mills AM, Longacre TA. Lynch syndrome screening in the gynecologic tract: current state of the art. Am J Surg Pathol. 2016;40:e35-44.

27. Goodfellow PJ, Billingsley CC, Lankes HA, Ali S, Cohn DE, Broaddus RJ, et al. Combined microsatellite instability, MLH1 methylation analysis, and immunohistochemistry for Lynch syndrome screening in endometrial cancers from GOG210: an NRG Oncology and Gynecologic Oncology Group Study. J Clin Oncol. 2015;33:4301-8.

28. Frampton GM, Fichtenholtz A, Otto GA, Wang K, Downing SR, He J, et al. Development and validation of a clinical cancer genomic profiling test based on massively parallel DNA sequencing. Nat Biotechnol. 2013;31:1023-31.
29. Pritchard CC, Salipante SJ, Koehler K, Smith C, Scroggins S, Wood B, et al. Validation and implementation of targeted capture and sequencing for the detection of actionable mutation, copy number variation, and gene rearrangement in clinical cancer specimens. J Mol Diagn. 2014;16:56-67.

30. Cheng DT, Mitchell TN, Zehir A, Shah RH, Benayed R, Syed A, et al. Memorial Sloan Kettering-Integrated Mutation Profiling of Actionable Cancer Targets (MSK-IMPACT): a hybridization capture-based next-generation sequencing clinical assay for solid tumor molecular oncology. J Mol Diagn. 2015;17:251-64.

31. Barroilhet L, Matulonis U. The NCI-MATCH trial and precision medicine in gynecologic cancers. Gynecol Oncol. 2018;148:585-90.

32. Sholl LM, Do K, Shivdasani P, Cerami E, Dubuc AM, Kuo FC, et al. Institutional implementation of clinical tumor profiling on an unselected cancer population. JCI Insight. 2016;1:e87062.

33. Nagarajan R, Bartley AN, Bridge JA, Jennings LJ, Kamel-Reid S, Kim A, et al. A window into clinical next-generation sequencingbased oncology testing practices. Arch Pathol Lab Med. 2017;141:1679-85.

34. Hendriks YMC, Wagner A, Morreau H, Menko F, Stormorken A, Quehenberger F, et al. Cancer risk in hereditary nonpolyposis colorectal cancer due to MSH6 mutations: impact on counseling and surveillance. Gastroenterology. 2004;127:17-25.

35. Berends MJW, Wu Y, Sijmons RH, Mensink RGJ, van der Sluis T, Hordijk-Hos JM, et al. Molecular and clinical characteristics of MSH6 variants: an analysis of 25 index carriers of a germline variant. Am J Hum Genet. 2002;70:26-37.

36. Gurin CC, Federici MG, Kang L, Boyd J. Causes and consequences of microsatellite instability in endometrial carcinoma. Cancer Res. 1999;59:462-6.

37. Duval A, Reperant M, Compoint A, Seruca R, Ranzani GN, Iacopetta $\mathrm{B}$, et al. Target gene mutation profile differs between gastrointestinal and endometrial tumors with mismatch repair deficiency. Cancer Res. 2002;62:1609-12.

38. Hause RJ, Pritchard CC, Shendure J, Salipante SJ. Classification and characterization of microsatellite instability across 18 cancer types. Nat Med. 2016;22:1342-50. 